\title{
CURRENT STATUS OF THE SECOND CAPE PHOTOGRAPHIC CATALOGUE
}

\author{
Chr. de Vegt \& N. Zacharias \\ Hamburger Sternwarte \\ Gojenbergsweg 112 \\ 2050 Hamburg 80 \\ Federal Republic of Germany \\ Margaret J. Penston \& C. A. Murray \\ Royal Greenwich Observatory \\ Herstmonceux Castle \\ Hail sham \\ East Sussex BN27 1RP \\ Engl and
}

\section{INTRODUCTION}

Photography of the southern hemisphere for the Second Cape Photographic Catalogue (CPC2) was commenced in 1962 by $R$ H Stoy, HM Astronomer at the Cape. During the following ten years nearly 6000 plates, each having two exposures and covering $4^{\circ} \times 4^{\circ}$ with fourfold corner to centre overlap were obtained. All plates have been measured on the GALAXY measuring machine at the Royal Greenwich Observatory under the general direction of W Nicholson. A preliminary catalogue giving positions and magnitudes of more than 50000 stars in the Cape Astrographic zone $\left(-40^{\circ}\right.$ to $\left.-52^{\circ}\right)$ has already been published (Nicholson et al. 1984), together with details of the programme. Measurement of the plates, initial identification of star i mages appearing on all overlapping plates, and photographic photometry have been carried out at RGO; the subseqent astrometric data reduction is being performed at Hamburg Observatory.

\section{PHOTOMETRY}

The spectral bandpass of the photographs is approximately 5300A-6400A, which is close to the Johnson $V$-band. In order to calibrate the image diameter measurements, photometric standards were derived from the literature, notably the CSI catalogue of the CDS Strasbourg and the Perth 70 catalogue (Hog \& von der Heide, 1976).

General statistics of the programme are given in Table I, in which we give for each declination zone, (1) the number of plates, (2) the total number of stars and (3) the number of photometric standards used for cal ibration. 
TABLE I

\begin{tabular}{rrrr}
\hline Zone & Plates & Stars & Stds. \\
\hline $0^{\circ}$ to $-20^{\circ}$ & 1980 & 99104 & 52085 \\
$-20^{\circ}$ to $-40^{\circ}$ & 1844 & 101217 & 56956 \\
$-40^{\circ}$ to $-52^{\circ}$ & 1008 & 51018 & 8100 \\
$-52^{\circ}$ to $-80^{\circ}$ & 1244 & 64269 & 38864 \\
$-80^{\circ}$ to $-90^{\circ}$ & 248 & 5120 & 3243 \\
\hline
\end{tabular}

The polar cap from $-80^{\circ}$ to $-90^{\circ}$ was photographed both above and below the pole.

Initially, each plate was calibrated using $V$ magnitudes of all available standards, omitting known variable stars and close doubles. The average magnitude for each programme star, over all the plates on which it was measured, was then adopted for a second calibration. The rms residuals from a quadratic calibration curve in this second approximation were of the order of $0.1 \mathrm{mag}$ or less for a single plate. Because of the deviation of the bandpass of the plates from the Johnson $V$ system, there are systematic differences of up to $0.3 \mathrm{mag}$ for the extreme reddest (too bright) and bluest (too faint) stars: this could not be corrected as the colours of most of the stars are unknown. It was not possible to find a single calibration curve to represent all plates. The photometric calibrations are now completed.

\section{CLASSICAL ASTROMETRIC REDUCTIONS}

Extensive software for classical single plate adjustment, block adjustment, analysis of accuracy properties and diagnostics has been developed by the astrometry group at the Hamburg Observatory. Using apparent places, including full allowance for refraction, the plate reduction model is for the first time definitely restricted to describe the metric properties of the plate and measuring machine, and the projection properties of the camera lens. From the investigation of various plate models in pilot samples of plates, the third order radial distortion of the lens has been found to be $+0 " .04 / \mathrm{deg}^{3}$.

Classical adjustments have been made for each plate using an eight parameter model, including orthogonal and non-orthogonal linear terms, and plate tilt terms $P, Q$ in RA and Dec respectively; the below pole plates in the polar cap appeared to be of lower astrometric quality and were not used. In the top panel of Fig.1 we show the variation with declination of plate scale, in parts per million (ppm), for the plates 
centred on the celestial meridian $R A=18 \mathrm{~h}$. Note that the Cape Zone (Dec $-40^{\circ}$ to $-52^{\circ}$ ) was photographed first, from 1962-64, after which the camera was dismounted. The remaining two panels illustrate the variations along the same meridian of the tilt terms $Q$ and $P$ respectively. The constancy of $P$ is very satisfactory. The somewhat 1 arger variation in $Q$ reflects adjustments to the camera during the course of the programme and the imprecise definition of the tangential point by marks imposed on the plates, as well as tube flexure. As already remarked, the Cape Zone was photographed first; this was followed between 1966 and 1967 by the zone $-30^{\circ}$ to $-40^{\circ}$. The remaining zones were photographed concurrently from 1968-72. Each plotted point in each diagram represents the mean of four plates.

In Fig. 2 we show the plate constants derived for the zone $0^{\circ}$ to $-20^{\circ}$ as functions of RA. Each point represents the mean of sixteen exposures. The two left-hand panels show the non-orthogonality (upper) and difference of scale (lower) of the axes of the GALAXY machine. The two right-hand panels give the tilt terms $Q$ (upper) and $P$ (lower).

All these diagrams illustrate the generally satisfactory representation of the geometric properties of the plates and GALAXY by the adjustment model.

\section{FUTURE WORK}

It is intended that the final catalogue will be based on a rigorous block adjustment of the whole series of plates. This will only be possible when the definitive SRS catalogue becomes available. It is expected that the positional precision of the final catalogue will be about $0 " .06$ at the epoch of observation.

It is now 15 years since the last plates were obtained. The positions for the current epoch will therefore be degraded by proper motions unless these can be derived. It will be recalled that for the provisional catalogue for the Cape Zone (Nicholson et al. 1984), proper motions for the stars common to the SAO catalogue were derived by comparison of our new positions with those at the epoch of SAO. It is proposed that, as an interim measure, a similar procedure will be followed for the remaining zones, thus giving an improved reference frame of SAO stars in the southern hemi sphere.

The results of a block adjustment of the provisional catalogue for the Cape Zone are described by one of us (NZ) elsewhere in this volume.

\section{REFERENCES}

Hog, E. \& von der Heide, J., 1976. Abh. Hamburger-Sternwarte, Band IX. Nichol son, W., Penston, Margaret J., Murray, C.A. \& de Vegt, Chr., 1984. Mon. Not. R. astr. Soc. 208, 911. 

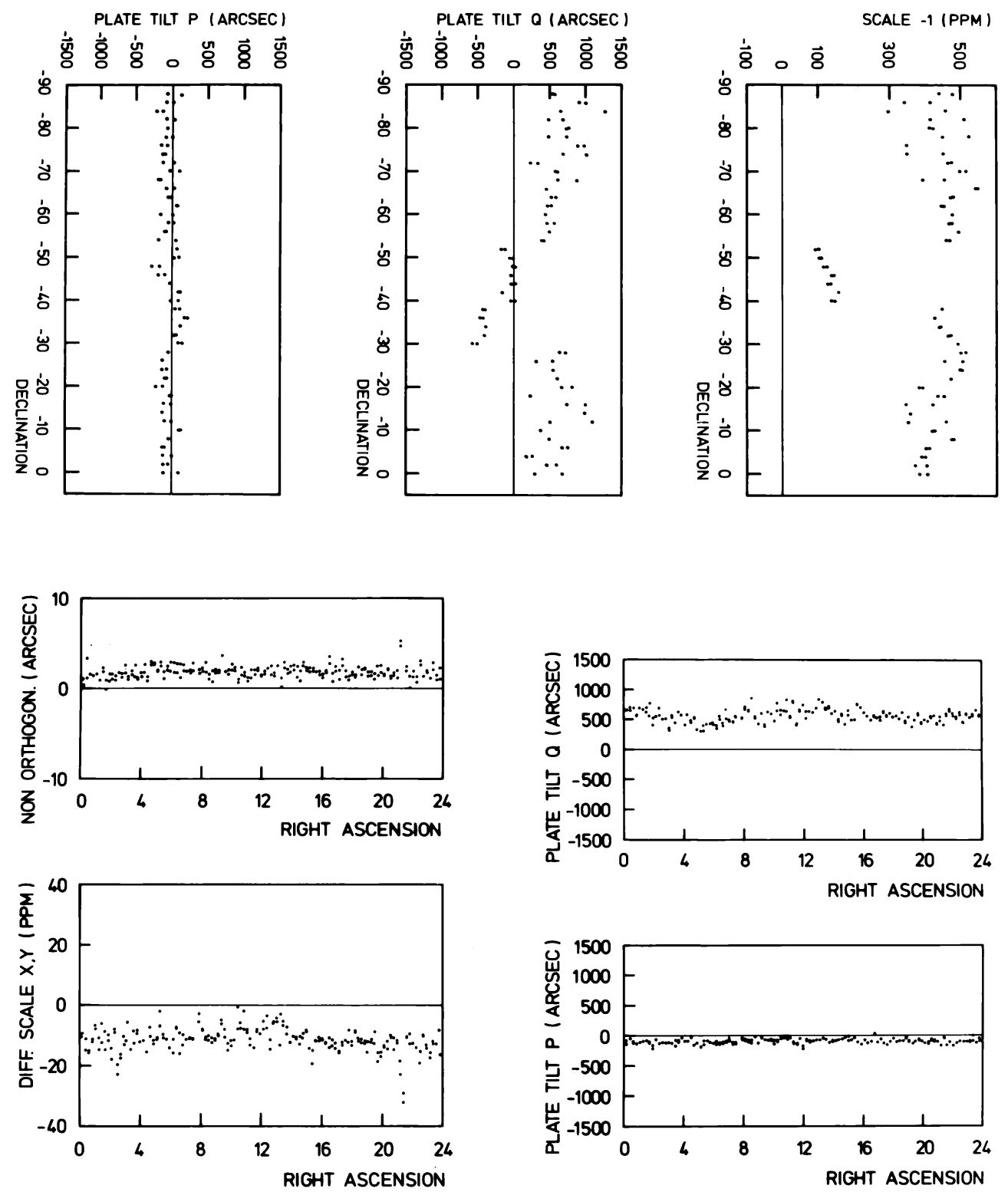

Fig 1 (upper): plate constants of all CPC2 plates along declination

Fig 2 (lower): plate constants of Cape Astrographic zone plates along right ascension 
Discussion:

\section{LASKER}

The photometric calibrations are

very interesting to other programs. Can you comment further on the sequences, particularly with respect to numbers of stars and precision? Also, when might this material become available?

MURRAY

The majority of photoelectric standards are for SRS stars which were published in the Perth 70 catalogue. The CSI was searched for any other available standards. This work has been carried out by Dr. Margaret Penston, who can supply you with further details. 